\title{
Metabolic Engineering of Oleaginous Yeast Rhodotorula toruloides for Overproduction of Triacetic Acid Lactone
}

\author{
Mingfeng $\mathrm{Cao}^{1}$, Vinh Tran ${ }^{1}$, Jiansong Qin ${ }^{2}$, Andrew Olson ${ }^{2}$, John Schultz ${ }^{1}$, Chunshuai \\ Huang $^{1}$, DONGMING XIE ${ }^{2}$, and Huimin Zhao ${ }^{1}$ \\ ${ }^{1}$ University of Illinois Urbana-Champaign Department of Chemical and Biomolecular \\ Engineering \\ ${ }^{2}$ University of Massachusetts Lowell
}

February 27, 2022

\begin{abstract}
The plant-sourced polyketide triacetic acid lactone (TAL) has been recognized as a promising platform chemical for the biorefinery industry. However, its practical application was rather limited due to low natural abundance and inefficient cell factories for biosynthesis. Here we report the metabolic engineering of oleaginous yeast Rhodotorula toruloides for TAL overproduction. We first introduced a 2-pyrone synthase gene from Gerbera hybrida ( GhPS) into R. toruloides and investigated the effects of different carbon sources on TAL production. We then systematically employed a variety of metabolic engineering strategies to increase the flux of acetyl-CoA by enhancing its biosynthetic pathways and disrupting its competing pathways. We found that overexpression of citrate lyase (ACL1) improved TAL production by $45 \%$ compared to the GhPS overexpressing strain, and additional overexpression of acetyl-CoA carboxylase (ACC1) further increased TAL production by $29 \%$. Finally, we characterized the resulting strain I12- $A C L 1$ - ACC1 using fed-batch bioreactor fermentation in glucose or oilcane juice medium with acetate supplementation and achieved a titer of $28 \mathrm{~g} / \mathrm{L}$ or $23 \mathrm{~g} / \mathrm{L}$ TAL, respectively. This study demonstrates that $R$. toruloides is a promising host for production of TAL and other acetyl-CoA-derived polyketides from low-cost carbon sources.
\end{abstract}

Submitted to Biotechnology and Bioengineering, 2-21-2022

Metabolic Engineering of Oleaginous Yeast Rhodotorula toruloides for Overproduction of Triacetic Acid Lactone

Mingfeng Cao ${ }^{\mathrm{a}}$, Vinh G. Tran ${ }^{\mathrm{a}}$, Jiansong Qin ${ }^{\mathrm{b}}$, Andrew Olson ${ }^{\mathrm{b}}$, J. Carl Schultz ${ }^{\mathrm{a}}$, Chunshuai Huang ${ }^{\mathrm{a}}$, Dongming Xie ${ }^{\mathrm{b}}$, Huimin Zhao ${ }^{\mathrm{a}, \mathrm{c},}{ }^{*}$

${ }^{a}$ Department of Chemical and Biomolecular Engineering, U.S. Department of Energy Center for Bioenergy and Bioproducts Innovation (CABBI), Carl R. Woese Institute for Genomic Biology, University of Illinois at Urbana-Champaign, Urbana, IL 61801, United States

b Department of Chemical Engineering, University of Massachusetts Lowell, Lowell, MA, 01854, USA

${ }^{c}$ Departments of Chemistry, Biochemistry, and Bioengineering, University of Illinois at Urbana-Champaign, Urbana, IL 61801, United States

Running title: Triacetic acid lactone production in Rhodotorula toruloides

${ }^{*}$ To whom correspondence should be addressed. Phone: (217) 333-2631. Fax: (217) 333-5052.

E-mail: zhao5@illinois.edu

\section{Graphical abstract}



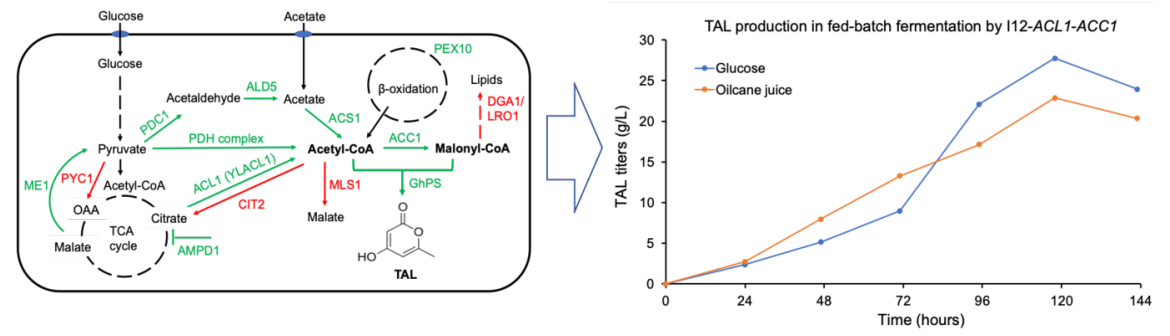

Triacetic acid lactone (TAL) is a promising platform chemical. Cao et al. overexpressed 2-pyrone synthase in oleaginous yeast Rhodotorula toruloides to produce TAL. They systematically evaluated various metabolic gene targets to increase acetyl-CoA and malonyl-CoA levels for TAL production and found that overexpression of both $A C L 1$ and $A C C 1$ led to $28 \mathrm{~g} / \mathrm{L}$ or $23 \mathrm{~g} / \mathrm{L}$ of TAL from glucose or oilcane juice with acetate supplementation, respectively, in fed-batch fermentation.

\section{Abstract}

The plant-sourced polyketide triacetic acid lactone (TAL) has been recognized as a promising platform chemical for the biorefinery industry. However, its practical application was rather limited due to low natural abundance and inefficient cell factories for biosynthesis. Here we report the metabolic engineering of oleaginous yeastRhodotorula toruloides for TAL overproduction. We first introduced a 2-pyrone synthase gene from Gerbera hybrida(GhPS ) into R. toruloides and investigated the effects of different carbon sources on TAL production. We then systematically employed a variety of metabolic engineering strategies to increase the flux of acetyl-CoA by enhancing its biosynthetic pathways and disrupting its competing pathways. We found that overexpression of citrate lyase (ACL1) improved TAL production by $45 \%$ compared to the GhPSoverexpressing strain, and additional overexpression of acetyl-CoA carboxylase (ACC1) further increased TAL production by $29 \%$. Finally, we characterized the resulting strain I12-ACL1-ACC1 using fedbatch bioreactor fermentation in glucose or oilcane juice medium with acetate supplementation and achieved a titer of $28 \mathrm{~g} / \mathrm{L}$ or $23 \mathrm{~g} / \mathrm{L}$ TAL, respectively. This study demonstrates that $R$. toruloides is a promising host for production of TAL and other acetyl-CoA-derived polyketides from low-cost carbon sources.

Keywords: Rhodotorula toruloides ; triacetic acid lactone; 2-pyrone synthase, metabolic targets; oilcane juice

\section{Introduction}

The demand for renewable biobased products has attracted great interest in using microbial cell factories to replace traditional petroleum-based chemical manufacturing processes (Cardenas \& Da Silva, 2014; Du et al., 2011; H. Liu et al., 2019; Markham et al., 2018). Among the chemicals that can be produced by microbial cell factories, polyketides represent a large family of naturally occurring metabolites with diversified industrial and biomedical applications. Due to the low yield by native producers and the challenge of introducing chiral centers by chemical catalysis, microbial bioconversion is considered a feasible approach for large-scale production of polyketides from renewable feedstocks (Keatinge-Clay, 2016; H. Liu et al., 2019; Robinson, 1991).

Triacetic acid lactone (TAL), also known as 4-hydroxy-6-methyl-2-pyrone, is a simple, yet interesting, polyketide. It has been demonstrated as a potential platform chemical for the production of commercially valuable bifunctional chemical intermediates and end products, including phloroglucinol (Zha et al., 2004), acetylacetone (Saunders et al., 2015), and sorbic acid (Chia et al., 2012). TAL is currently produced by chemical catalysis starting with the pyrolysis of acetic acid (Saunders et al., 2015). However, its industrial application was hampered by the detrimental catalysts and toxic byproducts (H. Liu et al., 2019). Therefore, it is highly desirable to develop an environment-friendly biological route for converting inexpensive substrates to TAL.

The biosynthesis of TAL is catalyzed by a type III polyketide synthase, 2-pyrone synthase (2-PS) via two iterative decarboxylation/condensation reactions using a starter acetyl-CoA and two extender malonyl-CoA 
molecules. 2-PS encoded by g2ps1 gene was first isolated fromGerbera hybrida (Abe et al., 2005; Eckermann et al., 1998), and has been genetically introduced to conventional organismsEscherichia coli (Li et al., 2018; Tang et al., 2013; Xie et al., 2006) and Saccharomyces cerevisiae (Cardenas \& Da Silva, 2014, 2016; Saunders et al., 2015; Sun et al., 2021) for TAL production. However, low titers were obtained in these conventional organisms due to cellular toxicity, limited intracellular acetyl-CoA pool, or unbalanced energy/cofactor supply (H. Liu et al., 2019).

Recent studies showed that oleaginous, nonconventional yeasts such as Yarrowia lipolytica and Rhodotorula toruloides (also known as Rhodosporidium toruloides ) can be used for efficient TAL production due to their potential high flux through the key polyketide precursors, acetyl-CoA, and malonyl-CoA (Abdel-Mawgoud et al., 2018; Park et al., 2018). As a well-known lipid producer, $Y$.lipolytica was chosen for TAL biosynthesis via heterologous expression of 2-pyrone synthase (Yu et al., 2018), and the best engineered Y. lipolytica strain achieved a titer of $35.9 \mathrm{~g} / \mathrm{L}$ TAL in 280 hours and a yield of up to $43 \%$ of the theoretical yield from glucose (Markham et al., 2018). $R$. toruloides is an oleaginous basidiomycete yeast, which can grow on various sugars and produce a broad range of lipid and nonlipid chemicals (Jagtap \& Rao, 2018; Zhang et al., 2016; Zhang et al., 2021). Compared with Y. lipolytica ,R. toruloides has a greater substrate range and natively produces TAG at much higher titers (Jagtap \& Rao, 2018; Zhang et al., 2016; Zhang et al., 2021), but was less explored as a result of the unannotated genome sequence (Coradetti et al., 2018; Zhu et al., 2012) and lack of sophisticated genetic tools (Park et al., 2018). Nevertheless, the recent progress on characterization of constitutive promoters (Nora et al., 2019; Wang et al., 2016), development of CRISPR based genome editing tools (Jiao et al., 2019; Otoupal et al., 2019; Schultz et al., 2019), RNA interference tool (X. Liu et al., 2019), genome-scale model (Dinh et al., 2019), and functional genomics (Coradetti et al., 2018) enable us to perform metabolic engineering of $R$. toruloides for production of value-added compounds (Wen et al., 2020), specifically TAL.

In this study, we first expressed codon-optimized 2-PS genes from various organisms in $R$. toruloides and investigated the production of TAL under different culture conditions. We then created and characterized a broad set of TAL-producing overexpression and knockout gene targets in $R$. toruloides IFO0880. After combinatorial optimization of various targets, our final strain of I12-ACL1-ACC1 achieved a maximum titer of $28 \mathrm{~g} / \mathrm{L}$ within 120 hours in fed-batch fermentation from glucose with acetate addition. Then, we demonstrated the feasibility of olicane juice, an inexpensive carbon source as the substrate for TAL production, which produced $23 \mathrm{~g} / \mathrm{L}$ TAL in fed-batch fermentation. This work not only establishes $R$. toruloides as a novel host organism for TAL biosynthesis but also demonstrates its potential as a biotechnological chassis for production of high-value chemicals from low-cost substrates.

\section{Materials and methods}

\section{Strains, media, and chemicals}

All strains used in this study are listed in Table 1. E. coliDH5 $\alpha$ (New England Biolabs, Ipswich, MA) was used to maintain and amplify plasmids, and cells were grown in Luria Broth (LB) medium at $37{ }^{\circ} \mathrm{C}$, $250 \mathrm{rpm}$ with $100 \mu \mathrm{g} / \mathrm{mL}$ ampicillin or $50 \mu \mathrm{g} / \mathrm{mL}$ kanamycin. $R$. toruloides IFO0880 and its mutants were grown at $30{ }^{\circ} \mathrm{C}, 250 \mathrm{rpm}$ in YPD media (1\% yeast extract, $2 \%$ peptone, $2 \%$ glucose) for routine handling. For selection or maintenance of transformants, $200 \mu \mathrm{g} / \mathrm{mL}$ G418 (KSE Scientific, Durham, NC), $50 \mu \mathrm{g} / \mathrm{mL}$ hygromycin (InvivoGen, San Diego, CA) or $100 \mu \mathrm{g} / \mathrm{mL}$ nourseothricin (Gold Biotechnology, St. Louis, MO) was supplemented as necessary. TAL production media include YPD, YP2D (1\% yeast extract, $2 \%$ peptone, $4 \%$ glucose), YPX (YP plus $2 \%$ xylose), YPDX (YP plus $1.4 \%$ glucose, $0.6 \%$ xylose), YPG (YP plus $2 \%$ glycerol), YPS (YP plus $2 \%$ sucrose), YP-NaAc (YP plus $2 \%$ sodium acetate) and SC (synthetic complete medium: $1.7 \mathrm{~g} / \mathrm{L}$ yeast nitrogen base, $5 \mathrm{~g} / \mathrm{L}$ ammonium sulfate, $0.78 \mathrm{~g} / \mathrm{L}$ complete synthetic mixture, $2 \%$ glucose).

LB broth, bacteriological grade agar, yeast extract, peptone, yeast nitrogen base, ammonium sulfate, and D-xylose were obtained from Difco (BD, Sparks, MD), while complete synthetic medium was purchased from MP Biomedicals (Solon, OH). TAL standard was purchased from Aldrich Chemical Co. (Milwaukee, 
WI). All restriction endonucleases, Q5 DNA polymerase, and Gibson Assembly Cloning Kit were purchased from New England Biolabs (Ipswich, MA). The QIAprep spin mini-prep kit was purchased from Qiagen (Valencia, CA), the Wizard Genomic DNA Purification Kit was purchased from Promega (Madison, WI), whereas Zymoclean Gel DNA Recovery Kit and Zymoprep Yeast Plasmid Miniprep Kits were purchased from Zymo Research (Irvine, CA). All other chemicals and consumables were purchased from Sigma (St. Louis, MO), VWR (Radnor, PA), and Fisher Scientific (Pittsburgh, PA). Sequences for key primers, N20 of SgRNA, and gene targets (Coradetti et al., 2018) were summarized in Table S1 and Table S2. Primers were synthesized by Integrated DNA Technologies (IDT, Coralville, IA), while heterologous genes were codon optimized by GeneOptimizer or the JGI BOOST tool and synthesized by GeneArt (Invitrogen, CA) or Twist Bioscience (San Francisco, CA). gRNAs were designed using the CRISPRdirect (https://crispr.dbcls.jp/) or the Benchling gRNA tool. DNA sequencing was performed by ACGT, Inc. (Wheeling, IL). Plasmid mapping and sequencing alignments were carried out using SnapGene software (GSL Biotech, available at snapgene.com).

\section{Plasmid construction}

Plasmids for 2-PS expression. The codon-optimized 2-PS genes were synthesized with two homologous ends to Mfe I and Spe I digested pGI2 (Abbott et al., 2013; Zhang et al., 2016) backbone, which contains nourseothricin resistance $\left(N A T^{\mathrm{R}}\right)$ for yeast, and then assembled with $p T E F 1$ promoter and T35Sterminator for gene expression by Gibson assembly in E. coli(Gibson et al., 2009).

Plasmids for overexpression of metabolic gene targets. The plasmid pRTG2-X (X represents gene expression targets) for gene targets expression was constructed based on a previously developed plasmid pRTN, which contains the $E$. coli genetic elements of pUC19 (pMB1 origin, ampicillin resistance), the $S$. cerevisiae genetic elements of pRS426 ( $2 \mu$ origin and URA3 selection marker), the strong $R$. toruloides $p 17$ or $p A N T$ promoter, the target gene, the T35S terminator, and a $R$. toruloides $\mathrm{G} 418$ resistance $\left(G 418^{\mathrm{R}}\right)$ cassette from NM9 (Schultz et al., 2019) using DNA assembler (Shao et al., 2009). The multiple gene expression plasmid pRTHyg-X was pieced together from NM8 (Schultz et al., 2019) for hygromycin resistance $\left(H y g{ }^{\mathrm{R}}\right), p A N T$ promoter and Tncbt for gene expression.

Plasmids for gene target knockout. The previously constructed plasmid pRTH-X (X represents gene knockout targets) was used for gRNA cloning and expression, which contains the E. coli genetic elements (pMB1 origin, ampicillin resistance), the $S$. cerevisiaegenetic elements of pRS426 ( $2 \mu$ origin and URA3), a gRNA expression cassette with the IFO0880 $5 \mathrm{~S}$ rRNA, tRNA ${ }^{\text {Tyr }}$, N20 (targeting the first $10 \%$ ORF), the $S$. cerevisiae SUP4 terminator, and a $R$. toruloides Hyg ${ }^{\mathrm{R}}$ cassette from pZPK-PGPD-HYG-Tnos (Lin et al., 2014). For multiple gene knockout, the plasmid pRT-Cas9-SgRNA-Hyg was constructed from pRTH-X with an integrated Cas9 expression cassette.

\section{Yeast transformation}

Most of the linear fragments for $R$. toruloidestransformation were generated by PCR amplification of the genes or gRNA expression cassettes, together with the selection markers using the primers ZPK F/R, or gRNA F/R (Table S1), respectively, whereas the fragments with sizes larger than $8 \mathrm{~kb}$ (e.g., ACC1-G418/Hyg ) were excised from the plasmids by restriction enzyme digestion. Fragments were then cleaned using DNA Clean \& Concentrator-5 Kit (Zymo Research) before transforming to R. toruloides .

$R$. toruloides was transformed using heat shock as previously described (Otoupal et al., 2019; Schultz et al., 2021). Briefly, a single colony was picked and cultured overnight at $30{ }^{\circ} \mathrm{C}$ in $3 \mathrm{~mL}$ YPD medium supplemented with an appropriate antibiotic if required. The overnight culture was transferred to $50 \mathrm{~mL}$ fresh YPD with an $\mathrm{OD}_{600}$ of 0.2 and cultured for another 4 hours at $30{ }^{\circ} \mathrm{C}$ to an $\mathrm{OD}_{600}$ of approximately 1.0. Cells were collected by centrifugation, washed twice with sterile water and once with $100 \mathrm{mM} \mathrm{LiAc}(\mathrm{pH}$ 7.6) (Sigma Aldrich, St. Louis, MO), and then resuspended in a transformation mixture of $240 \mu \mathrm{L}$ PEG3350 (Sigma Aldrich, St. Louis, MO), $36 \mu \mathrm{L} 1 \mathrm{M}$ lithium acetate, $50 \mu \mathrm{L}$ of $2 \mathrm{mg} / \mathrm{mL}$ salmon sperm DNA (Sigma Aldrich, St. Louis, MO), and 1-2 $\mu \mathrm{g}$ of linear DNA dissolved in $34 \mu \mathrm{L}$ of water. The cells were incubated with $200 \mathrm{rpm}$ shaking in the mixture for $30 \mathrm{~min}$ at $30{ }^{\circ} \mathrm{C}$. Then, $34 \mu \mathrm{L}$ of dimethyl sulfoxide (DMSO) was 
added to the mixture, which was briefly vortexed, and heat shocked at $42{ }^{\circ} \mathrm{C}$ for $20 \mathrm{~min}$. The cells were pelleted, washed with YPD, resuspended in $2 \mathrm{~mL} \mathrm{YPD,} \mathrm{and} \mathrm{recovered} \mathrm{overnight} \mathrm{with} \mathrm{shaking.} \mathrm{Cells} \mathrm{were}$ then collected and plated on YPD solid medium supplemented with the appropriate antibiotic(s). To verify the integrated fragments, genomic DNA was extracted using the Wizard Genomic DNA Purification Kit (Promega), and the target locus was PCR amplified for sequencing.

Culture tube or shake flask fermentationSingle colonies were picked and cultured for 24 hours at $30{ }^{\circ} \mathrm{C}$ in $3 \mathrm{~mL}$ YPD liquid medium supplemented with appropriate antibiotics in $14 \mathrm{~mL}$ culture tubes (VWR) as seed culture. Fermentations were inoculated from seed culture to media with alternative carbon sources at an initial $\mathrm{OD}_{600}$ of 0.2 and grown for an additional 72 hours prior to sample preparation. For acetate spike, filter sterilized 20x sodium acetate (NaAc) was added to media at $12 \mathrm{~h}$. Samples were collected by centrifuge and diluted 20 times for high performance liquid chromatography (HPLC) analysis. Since a deficient cell growth and tiny amounts of TAL were obtained in YP (1\% yeast extract and $2 \%$ peptone) medium, yield was calculated based on the produced TAL over all carbons of sugars and/or acetate.

\section{Fed-batch fermentation}

For fed-batch fermentation in bioreactors, single colonies of $R$. toruloides I12-ACL1 and I12-ACL1 -ACC1 were used to inoculate shake flask cultures with each containing $50 \mathrm{~mL}$ of YPAD medium supplemented with appropriate antibiotics, as described in section 2.1. Cells in the flask cultures were grown at $30{ }^{\circ} \mathrm{C}$ and 250 $\mathrm{rpm}$ until the $\mathrm{OD}_{600}$ reached 2-5. The seed culture from each flask was transferred to a 1-L bioreactor (Biostat B-DCU, Sartorius, Germany) with $0.7 \mathrm{~L}$ fermentation medium, which contained $15 \mathrm{~g} / \mathrm{L}$ yeast extract, $15 \mathrm{~g} / \mathrm{L}$ peptone, $10 \mathrm{~g} / \mathrm{L}\left(\mathrm{NH}_{4}\right)_{2} \mathrm{SO}_{4}, 6 \mathrm{~g} / \mathrm{L} \mathrm{KH}_{2} \mathrm{PO}_{4}, 2 \mathrm{~g} / \mathrm{L} \mathrm{Na}{ }_{2} \mathrm{HPO}_{4}, 1 \mathrm{~mL} / \mathrm{L}$ Antifoam 204 (Sigma-Aldrich), 1.5 $\mathrm{mg} / \mathrm{L}$ Thiamin $\cdot \mathrm{HCl}, 1.2 \mathrm{~g} / \mathrm{L} \mathrm{MgSO}_{4}, 2 \mathrm{~mL} / \mathrm{L}$ trace metals $(100 \mathrm{X}), 50 \mathrm{~g} / \mathrm{L}$ glucose, and appropriate antibiotics (same as described in section 2.1). The trace metal $(100 \mathrm{X})$ solution contained $10 \mathrm{~g} / \mathrm{L}$ citric acid, $1.5 \mathrm{~g} / \mathrm{L}$ $\mathrm{CaCl}_{2} \cdot 2 \mathrm{H}_{2} \mathrm{O} .10 \mathrm{~g} / \mathrm{L} \mathrm{FeSO}{ }_{4} \cdot 7 \mathrm{H}_{2} \mathrm{O}, 0.39 \mathrm{~g} / \mathrm{L} \mathrm{ZnSO}_{4} \cdot 7 \mathrm{H}_{2} \mathrm{O}, 0.38 \mathrm{~g} / \mathrm{L} \mathrm{CuSO}_{4} \cdot 5 \mathrm{H}_{2} \mathrm{O}, 0.2 \mathrm{~g} / \mathrm{L} \mathrm{CoCl}_{2} \cdot 6 \mathrm{H}_{2} \mathrm{O}$, and $0.3 \mathrm{~g} / \mathrm{L} \mathrm{MnCl}_{2} \cdot 4 \mathrm{H}_{2} \mathrm{O}$. The dissolved oxygen level was maintained at $20 \%$ of air saturation, and the temperature was set at $30^{\circ} \mathrm{C}$. The $\mathrm{pH}$ value was controlled at 6.0 using glacial acetic acid and $10 \mathrm{M} \mathrm{KOH}$. When the residual glucose concentration decreased to nearly $0 \mathrm{~g} / \mathrm{L}$ (as indicated by a sharp decreasing in agitation speed and an increase in $\mathrm{pH}$ value), continuous feeding of glucose (from a $600 \mathrm{~g} / \mathrm{L}$ stock solution) was used to maintain its residual concentrations within $20 \mathrm{~g} / \mathrm{L}$. Sodium acetate $(460 \mathrm{~g} / \mathrm{L})$ was fed to the fermenter in pulse at different time points: $10 \mathrm{~mL}$ at 36, 48,60, 72, and 96 hours, and $8 \mathrm{~mL}$ at 108, 120, 132, 144, 156, and 168 hours, respectively. The fed-batch fermentation was conducted in biological duplicates.

For the fed-batch fermentation experiments with oilcane, the original oilcane feed solution containing a total sugar of $152 \mathrm{~g} / \mathrm{L}(68.9 \mathrm{~g} / \mathrm{L}$ glucose, $61.6 \mathrm{~g} / \mathrm{L}$ fructose, and $21.5 \mathrm{~g} / \mathrm{L}$ sucrose $)$ was concentrated to about $450 \mathrm{~g} / \mathrm{L}$ total sugar by evaporation through the boiling at atmospheric pressure. The concentrated oilcane feed solution was further autoclaved at $121{ }^{\circ} \mathrm{C}$ for $30 \mathrm{~min}$ before it was used to provide initial sugars in the medium and to feed sugars during the fed-batch fermentation. The initial medium contained a total oilcane sugar of $50 \mathrm{~g} / \mathrm{L}$ and all other medium components, as described previously for the glucose fed-batch fermentation. The oilcane feeding started when the initial sugars were depleted, as indicated by a sharp decrease in agitation speed and an increase in $\mathrm{pH}$ value. Oilcane was fed to control the residual glucose concentrations within $0^{\sim} 10 \mathrm{~g} / \mathrm{L}$. All other fermentation conditions, including acetate feeding, were same as previously described for the fed-batch fermentation experiments with glucose.

\section{Analytical methods}

Samples were prepared by diluting in methanol to the linear range, vortex mixing, and centrifuging at $16,000 \mathrm{~g}$ for $5 \mathrm{~min}$ to remove cells. After being filtered by a $0.2 \mu \mathrm{m}$ filter, the supernatant was injected into the HPLC for TAL, sugars, and acetate analyses. (1) TAL characterization . The analytical HPLC was carried out on an Agilent 1260 Infinity series instrument equipped with a diode array detector (DAD) using a Phenomenex Kinetex ${ }^{\circledR} 5$ um EVO C18 $100 \AA$ LC column $(150 \times 4.6 \mathrm{~mm}$; Phenomenex, USA). The solvent system comprises solvent A (water supplemented with $0.1 \%$ trifluoroacetic acid) and B (acetonitrile supplemented with $0.1 \%$ trifluoroacetic acid). The elution process runs the following program: $2 \% \mathrm{~B}$ to $7 \% \mathrm{~B}$ 
(linear gradient, 0-5 min), 7\% B to $95 \%$ B (5-6 min), 95\% B (isocratic elution, 6-8 min), $95 \%$ B to $2 \%$ B (8-9 $\min$ ), $2 \%$ B (isocratic elution, 9-11 min). Full wavelength scanning (UV/Vis) and Liquid ChromatographyMass Spectrometry (LC-MS) were performed to determine the specific absorbance and molecular weight of the target products using $>98.0 \%$ purity TAL as a reference. LC-MS analysis was running on a Waters Synapt G2-Si ESI/LC-MS (Milford, MA), equipped with ESI positive ion mode (Bruker, Amazon SL Ion Trap) and a Kinetex 2.6- $\mu \mathrm{m}$ XB-C18 $100 \AA$ (Phenomenex). (2) Sugar and acetate analyses.Glucose, xylose, glycerol, sucrose, and acetate consumptions were measured using an Agilent 1260 Infinity HPLC (Santa Clara, CA), equipped with Rezex ${ }^{\mathrm{TM}}$ ROA-Organic Acid $\mathrm{H}^{+}(8 \%)$ column (Phenomenex Inc., Torrance, CA) and a refractive index detector (RID). The column and detector were run at $50 \mathrm{degC}$ and $0.6 \mathrm{~mL} / \mathrm{min}$ of $0.005 \mathrm{~N} \mathrm{H}_{2} \mathrm{SO}_{4}$ was used as the mobile phase (J.-J. Liu et al., 2019).

\section{Results and discussion}

\section{$R$. toruloides can serve as a TAL producer}

High lipid production in oleaginous organisms like $R$. toruloidessuggests a great potential for these organisms to synthesize alternative acetyl-CoA-derived products, specifically type III polyketide, TAL (Markham et al., 2018; Park et al., 2018; Wen et al., 2020). It was reported that $R$. toruloides could grow normally under harsh conditions (Lyu et al., 2021) or cultures with non-native products, including fatty alcohols (Liu et al., 2020), fatty acid ethyl esters (Zhang et al., 2021), and limonene (Liu et al., 2021). The product tolerance assay also showed that $R$. toruloides possessed a similar growth profile in YPD and YPD with $5 \mathrm{~g} / \mathrm{L}$ TAL but longer lag and log phases in YPD with $7 \mathrm{~g} / \mathrm{L}$ TAL supplementation (Fig. S1). Therefore, R. toruloides can be potentially engineered to produce high titers of TAL without significantly detrimental growth effects.

As the 2-pyrone synthase (2-PS) gene tested for TAL biosynthesis in yeast and bacteria was mainly from Gerbera hybrida (GhPS, Uniprot ID: P48391), we sought to explore additional 2-PS genes from a range of alternative organisms and characterize their function in $R$.toruloides . To assist the selection of 2-PS genes, the Enzyme Function Initiative-Enzyme Similarity Tool (EFI-EST) was used to create the sequencesimilarity network (SSN) based on the InterPro family IPR011141 (Type-III polyketide synthase) (Gerlt et al., 2015).GhPS and three additional 2-PS genes from previously unexplored species, Vitis vinifera (Protein ID: CK203_022254),Sphaceloma murrayae (Protein ID: CAC42_3419), andAspergillus oryzae (Protein ID: AO090701000566) were codon-optimized using the most frequently used codon (Table S2), synthesized, and cloned to pGI2 under the $p T E F 1$ promoter. The PCR-amplified 2-PS - NAT cassettes were transformed and randomly genome-integrated via NHEJ to $R$. toruloides. However, of these genes, only GhPS produced TAL (Fig. S2). The strain I12 with one copy GhPS expression produced $2.0+-0.1 \mathrm{~g} / \mathrm{L}$ TAL in YPD medium at $72 \mathrm{~h}$ in a culture tube, which was close to that obtained from the $Y$. lipolytica strain $(2.1 \mathrm{~g} / \mathrm{L})$ with four copies of GhPS expression in a defined synthetic medium (Markham et al., 2018). Although codon optimization algorithms and genome integration loci may affect the GhPS expression, it indicates $R$. toruloides can serve as a promising platform for TAL production.

\section{TAL production using various substrates}

To evaluate the effects of substrates on TAL production, we chose the commonly used carbon sources, including xylose (X), glycerol (G), sucrose (S), complete synthetic medium (SC), and glucose/xylose mixed sugar (DX) (Fig. 1A). The results showed that YPD was a more preferred medium than SC, with a 4-fold higher TAL titer; compared to glucose, glycerol and glucose/xylose produced 5\% $10 \%$ higher TAL, while xylose and sucrose decreased TAL production. We also observed deficient cell growth and residual sugars in YPX, YPS, and SC media (Table S3), indicating a positive correlation between cell growth and TAL titer.

It has been demonstrated that acetate feeding was beneficial to acetyl-CoA supply and TAL biosynthesis in S. cerevisiae and Y. lipolytica (H. Liu et al., 2019; Markham et al., 2018; Sun et al., 2021). We therefore supplemented $0.5 \%, 1 \%$, and $2 \% \mathrm{NaAc}$ to YPD with the $12 \mathrm{~h} R$. toruloides cell culture and observed significant improvements, $67 \% \sim 80 \%$ higher TAL production under these spiking conditions, representing a similar titer to that of YP2D (3.6 g/L TAL from YP-4\% glucose at $72 \mathrm{~h})$ and $\sim 30 \%$ of the theoretical yield calculated from both glucose $(2 \%)$ and acetate $(0.5 \%)$ in culture tube (Fig. 1B). 
To explore the potential role of acetate during TAL biosynthesis, we provided $2 \% \mathrm{NaAc}$ as an alternative carbon source. However, the TAL produced from YP-NaAc was only $42 \%$ of that produced from YPD (Fig. 1B). The residual amounts of NaAc were also measured for the above-mentioned NaAc media, and only YPD $+0.5 \% \mathrm{NaAc}$ showed depletion of acetate after fermentation while a portion of acetate left in $\mathrm{YPD}+1 \% \mathrm{NaAc}, \mathrm{YPD}+2 \% \mathrm{NaAc}$, and YP-NaAc (Table S3). The acetate consumption indicated that acetate may not only act as a substrate for TAL production, but also be associated with the redox and regulatory mechanism, which has been elaborated in Y. lipolytica (Markham et al., 2018).

\section{Single gene target engineering to improve TAL production}

It is generally recognized that malonyl-CoA is the limiting precursor for polyketide synthase (Xu et al., 2011; Zha et al., 2009). Therefore, we overexpressed the endogenous acetyl-CoA-carboxylase (ACC1) to test whether the conversion of acetyl-CoA to malonyl-CoA would facilitate TAL synthesis (Fig. 2). As shown in Fig. 3A, overexpression of $A C C 1$ did not markedly enhance TAL production with only $6 \%$ improvement in YP2D at $120 \mathrm{~h}$ compared to that of the starting strain I12. To further drive the condensation of acetyl-CoA and malonyl-CoA, we introduced a second copy of GhPS gene via genome integration and achieved $4.8 \mathrm{~g} / \mathrm{L}$ TAL at $120 \mathrm{~h}, \sim 11 \%$ higher than that of I12 (Fig. 3A). Based on these results, we deduced that the limiting precursor for TAL overproduction was acetyl-CoA instead of malonyl-CoA. Therefore, we sought to increase the flux of acetyl-CoA by enhancing its biosynthetic pathways and disrupting its competing pathways.

(1) Enhancing acetyl-CoA biosynthetic pathways . We explored three distinct metabolic engineering strategies and characterized the roles of associated gene targets in TAL production (Fig. 3). First, we investigated the pyruvate dehydrogenase (PDH) complex pathway and overexpressed its subunits, E1 and E3 (LPD1) in I12 strain. Fermentation showed that both E1 and E3 overexpression improved TAL production by $16 \%$, which reached $\sim 5.3 \mathrm{~g} / \mathrm{L}$ at $120 \mathrm{~h}$ (Fig. 3A and Table S4). It is known that the PDH complex is located in the mitochondrial matrix in eukaryotes, and its compartmentalization is mediated via mitochondrial targeting sequence (MTS). However, the improved TAL production indicated that the tested subunits of E1 and E3 may not contain a fully featured MTS, or there may be leaky expression of these two subunits in the cytoplasm, which was similar to that of the overexpression of PDH complex in S. cerevisiae (Lian et al., 2014) and Y. lipoytica (Markham et al., 2018). As we failed to construct the mutants of other subunits of PDH, overexpression of the complete PDH or a cytoplastic PDH complex (i.e.,E. coli cytoPDH) (Cardenas \& Da Silva, 2016; Kozak et al., 2014) can be a potential strategy to increase the acetyl-CoA level.

Second, we evaluated the pyruvate dehydrogenase bypass pathway, which converts pyruvate to acetyl-CoA through a three-step reaction sequentially catalyzed by pyruvate decarboxylase (PDC), acetaldehyde dehydrogenase (ALD), and acetyl-CoA synthetase (ACS). The corresponding genes, PDC1, ALD5 , and ACS1 were individually overexpressed in I12 (Fig. 2). As shown in Fig. 3A and Table S4, overexpression of PDC1 and ALD5 improved TAL production by $13 \%$ and $21 \%$, reaching $5.1 \mathrm{~g} / \mathrm{L}$ and $5.5 \mathrm{~g} / \mathrm{L}$ in YP2D at $120 \mathrm{~h}$, respectively. However, no TAL improvement was achieved by ACS1 overexpression, which is similar to the study in $S$. cerevisiaewhere ACS1 overexpression did not improve $n$-butanol production because of low activity or post-translational deactivation (Lian et al., 2014).

Third, we explored the citrate route, a pathway that generates cytosolic acetyl-CoA from citrate and was reported to be present only in oleaginous yeasts (Pomraning et al., 2019; Vorapreeda et al., 2012; Zhu et al., 2012). The pathway gene ACL1 , encoding ATP-citrate lyase has been overexpressed to increase lipid production in Y. lipolytica (Blazeck et al., 2014; Wang et al., 2015). A multi-omic analysis of $R$. toruloides also revealed that $A C L 1$ was expressed at extremely high level during lipogenesis stage (Zhu et al., 2012). Therefore, the endogenous ACL1 was overexpressed in I12 strain (Fig. 2), and the TAL production was dramatically improved by $45 \%$, to $6.6 \mathrm{~g} / \mathrm{L}$ in YP2D in a test tube at $120 \mathrm{~h}$ (Fig. 3A and Table S4), which was $\sim 35 \%$ of the theoretical yield.

In addition, we overexpressed metabolic targets that could indirectly increase metabolic flux of acetyl-CoA, including AMPD1 (encoding AMP deaminase) (Zhang et al., 2019), ME1 (encoding malic enzyme),PEX10 (encoding peroxisomal matrix protein), and YLACL1(ACL1 from Y. lipolytica and sequence included in 
Table S2) (Blazeck et al., 2014) (Fig. 2). The results showed that PEX10and YLACL1 overexpression increased TAL titer by $12 \%$ and $11 \%$, respectively, whereas AMPD1 decreased TAL titer and ME1 had no effect on TAL production (Fig. 3A). This suggests that up-regulation of $\beta$-oxidation by enhancing peroxisome biogenesis through PEX10 overexpression is an alternative way to recycle acetyl-CoA for TAL production in R. toruloides .

(2) Disrupting acetyl-CoA competing pathways. Removing acetyl-CoA consuming pathways was demonstrated as an effective way to increase the availability of acetyl-CoA. In yeast, the glyoxylate shunt allows acetyl-CoA to be converted into a C4 carbon without carbon loss (Dolan \& Welch, 2018). Therefore, we performed the inhibition of two key reactions of the glyoxylate cycle, namely peroxisomal citrate synthase, encoded by CIT2 , and cytosolic malate synthase, encoded by $M L S 1$ (Chen et al., 2013), by a previously developed CRISPR/Cas9 method (Schultz et al., 2019). As shown in Fig. 3B, compared with I12-Cas9 strain, the deletion of CIT2 and MLS1 improved TAL production by $14 \%$ and $20 \%$, respectively.

In addition, we investigated the effects of disrupting other gene targets, including two acyltransferases (encoded by DGA1 / LRO1 ), pyruvate carboxylase (encoded by PYC1), serine esterase or patatin-domaincontaining protein (encoded by NTE1), and mitochondrial $\mathrm{NAD}^{+}$transporter (encoded by YIA6 ). Among them, DGA1 and LRO1 are involved in TAG formation in $Y$. lipolytica (Athenstaedt, 2011), and PYC1 , NTE1 , and YIA6 were reported to improve TAL production in $S$. cerevisiae (Cardenas \& Da Silva, 2014). The fermentation showed that the deletion of DGA1 and LRO1 improved TAL titer by $11 \%$ and $19 \%$, respectively, while the deletion of NTE1, YIA6 , and PYC1 had a marginal effect on TAL production (Fig. 3B), which is inconsistent with the observation in $S$. cerevisiae .

\section{Multiple gene target engineering to improve TAL production}

To further investigate the effects of multiple gene targets on TAL production in a combinatorial manner, we selected the top targets that improved TAL production more than $12 \%$, i.e., ACL1 , ALD5, MLS,

\section{Hosted file}

image2.emf available at https://authorea.com/users/462525/articles/557932-metabolicengineering-of-oleaginous-yeast-rhodotorula-toruloides-for-overproduction-of-triaceticacid-lactone

Fig. 1. TAL production in $R$. toruloides -I12 using different substrates. (A) Commonly used sugars; (B) Acetate spiking affects TAL production.

\section{Hosted file}

image3.emf available at https://authorea.com/users/462525/articles/557932-metabolicengineering-of-oleaginous-yeast-rhodotorula-toruloides-for-overproduction-of-triaceticacid-lactone

Fig. 2. Metabolic pathway engineering for TAL biosynthesis in $R$. toruloides . PDC1, pyruvate decarboxylase; ALD5, acetylaldehyde dehydrogenase; ACS1, acetyl-CoA synthetase ACS1, ACC1, acetyl-CoA carboxylase; GhPS, 2-pyrone synthase gene from Gerbera hybrida ; PDH, pyruvate dehydrogenase; ACL1, citrate lyase; YLACL1, citrate lyase from Yarrowia lipolytica ; AMPD1, AMP deaminase; ME1, malic enzyme; PEX10, peroxisomal matrix protein; PYC1, pyruvate carboxylase; MLS1, cytosolic malate synthase; CIT2, peroxisomal citrate synthase; DGA1, diacylglycerol acyltransferase; LRO1, lecithin cholesterol acyltransferase. Note: Some metabolites were not positioned following their intracellular compartmentalization.

\section{Hosted file}

image4.emf available at https://authorea.com/users/462525/articles/557932-metabolicengineering-of-oleaginous-yeast-rhodotorula-toruloides-for-overproduction-of-triaceticacid-lactone 
Fig. 3. TAL production from different metabolic engineering strategies. A. Overexpression of selected gene targets; B. Disruption of selected gene targets. C. Multiple gene targets by combinatorial engineering.

\section{Hosted file}

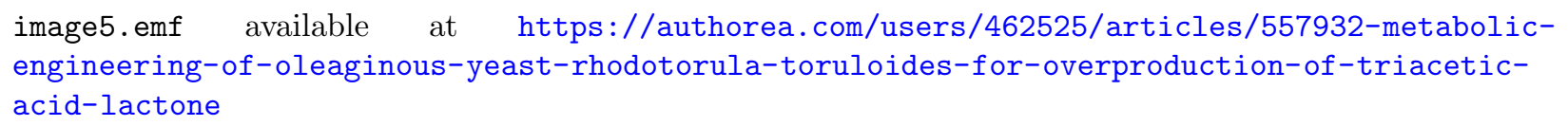

Fig. 4. Fed-batch bioreactor fermentation of $R$.toruloides . A. The cell growth $\left(\mathrm{OD}_{600}\right)$, total consumed glucose and acetate under glucose-based medium; B. The TAL titer and corresponding yield to its theoretical yield under glucose-based medium; $\mathrm{C}$. The cell growth $\left(\mathrm{OD}_{600}\right)$, total consumed glucose and acetate under oilcane juice-based medium; D. The TAL titer and corresponding yield to its theoretical yield under oilcane juice-based medium. 